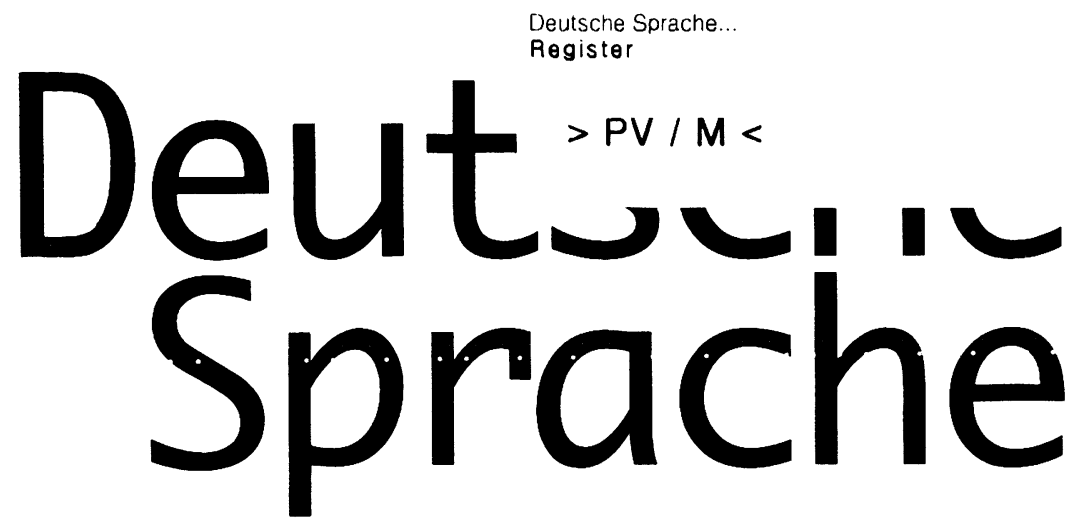

36. Jahrgang 2008

ZEITSCHRIFT

FÜ R

THEORIE

PRAXIS

DOKUMENTATION

Im Auftrag des

Instituts für deutsche Sprache, Mannheim

herausgegeben von

Ludwig M. Eichinger (Geschäftsführung),

Martine Dalmas, Reinhard Fiehler,

Ulla Fix, Gisela Zifonun

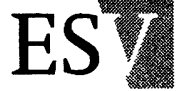




\section{JAHRESINHALTSVERZEICHNIS 2008}

Seite

Aufsätze

THEMENHEFT: ERKENNTNISSE VGM RANDE

ZUR INTERAKTION VON PROSODIE, INFORMATIONSSTRUKTUR, SYNTAX UND BEDEUTUNG

Eva Breindl (MANnheim)/Maria Thurmair (Regensburg)

Vorwort: Erkenntnisse vom Rande

Zur Interaktion von Prosodie, Informationsstruktur, Syntax und Bedeutung

27 EVA BREINdL (MANNHEIM)

Die Brigitte nun kann der Hans nicht ausstehen

Gebundene Topiks im Deutschen

50

WILHELM OPPENRIEDER (MÜNCHEN)

Statt eines Blumenstraußes

Überlegungen zu einer Partikel

KARIN PITTNer (Bochum)

Schlecht dran oder gut drauf?

Überlegungen zur Grammatikalisierung und Akzentuierung von

Pronominaladverbien

3 Beatrice Primus (KölN)

Diese - etwas vernachlässigte - pränominale Herausstellung

(Fortsetzung des Themenheftes)

MARIA ThURMAIR (REgENSBURG)

Mei, der Hans und die Brigitte!

Eine formale und funktionale Analyse der bayerischen Partikel mei

287 WeRnER ABRAHAM (MÜNCHEN)

Tempus- und Aspektkodierung als Textverketter:

Vorder- und Hintergrundierung

127 Lingling Chang (Nanjing/China)

Resultative Prädikate, Verbpartikeln und eine konstruktions-

grammatische Überlegung

220 ARNULF DEPPERMANN / REINHOLD SCHMITT (MANNHEIM)

Verstehensdokumentationen: Zur Phänomenologie von Verstehen in der Interaktion

305 ElKe Donalies (Mannheim)

Sandstrand, sandy beach, plage de sable, arenile, piaskowy plaza, homokos part Komposita, Derivate und Phraseme des Deutschen im europäischen Vergleich

114 Hilke Elsen (MünChen)

Kontaminationen im Randbereich der deutschen Grammatik 
246 Ulrike Freywald (Potsdam)

Zur Syntax und Funktion von dass-Sätzen mit Verbzweitstellung

341 Nader Haghani (Teheran)

Zur Entwicklung des Faches Deutsch als akademische Disziplin im Iran

193 LUDGER HOFFMANN (DORTMUND)

Über $j a$

176 STEFAN KüHTZ (HöHR-GRENZHAUSEN)

Phraseologie in Fachtexten: Funktionen und analytisches Potenzial

324 GÜler MUNGAN (Ziverbey-Istanbul)

Partikel- und Präfixsubstantiva

Eine analytische Fallstudie zu deren Morphologie und Semantik

146 BRITT-MARIE SCHUSTER (GIESSEN)

Verarmung oder Bereicherung der Schriftkultur?

Zur Beschreibung und Interpretation der Übergangsformen zwischen

Parataxe und Hypotaxe im gegenwärtigen Printjournalismus

\section{Dokumentationen}

356 Valentina CREstani (ToRINo)

Bericht von der dritten Tagung „Deutsche Sprachwissenschaft in Italien“

371 PATRICK Vosskamp (ESSEN)

Die Arbeit mit Transkripten in der Praxis

Bericht über das 40. Treffen des „Arbeitskreises Angewandte

Gesprächsforschung“ in Freiburg am 4. und 5. Mai 2007

\section{Besprechungen}

380 Petra BalsLiemke (WUPPERTAL)

Was sprichst du wo?

Zwei Sammelbände enthalten aktuelle Tendenzen der deutschen

Gegenwartssprache 


\title{
KONTAMINATIONEN IM RANDBEREICH DER DEUTSCHEN GRAMMATIK
}

\begin{abstract}
Kontaminationen befinden sich in einem peripheren Bereich der Wortbildung, das wird auch durch die unterschiedlichen Bezeichnungen und Strukturierungsversuche deutlich. Im Folgenden werden die Kontaminationen anderen Wortbildungstypen gegenübergestellt und einige aktuelle Beispiele aus neueren Veröffentlichungen aufgeführt, um ihre Rolle in unterschiedlichen Wortschatzbereichen zu betrachten. Anschließend soll der Versuch gemacht werden, die Kategorie Kontamination unter prototypischen $\mathrm{Ge}-$ sichtpunkten zu strukturieren. Der Artikel dient als Anstoß zu einer parole-orientierten Betrachtungsweise von Grammatik.

Blends belong to the periphery of German grammar, as is shown by the variety of different terms in use and the fruitless efforts to find stringent regularities in their structure. In this article, blends and other word-formations will be contrasted, and recent examples will be presented in order to examine the position of blends in a number of areas of the German lexicon. Subsequently, a prototypical structure of the category 'blending' is suggested as a first step towards an analysis of grammar on the basis of actual language use.
\end{abstract}

\section{Einleitung}

Als Alice in ihrem zweiten Abenteuer auf Humpty Dumpty trifft, muss sie sich zunächst an seine eigenwillige Sprache gewöhnen, weil er viele „schwierige“ Wörter gebraucht. Auf ihre Frage nach slithy erhält sie folgende Antwort: „Well, ,slithy' means , lithe and slimy.' ,Lithe' is the same as ,active'. You see it's like a portmanteau - there are two meanings packed up into one word" (Carroll 1976, S. 215). Das vielzitierte Beispiel entwickelte sich zur Erklärungsgrundlage und Basis für die spätere Bezeichnung solcher Wörter - Portmanteauwort, Kofferwort, mot valise. Andere Termini im Deutschen sind u. a. Wortverschmelzung, Wortmischung, Wortkreuzung, Mischwortbildung, Zusammenziehung, Kontamination oder Blending. Die Fülle der Namen weist bereits auf eine gewisse Verlegenheit bei der Behandlung dieser peripheren Erscheinung hin. Der nun folgende Artikel bringt einen Überblick über bisherige Ergebnisse und aktuelle Beispiele und versucht, für das Phänomen Kontamination einen Platz in der Wortbildung des Deutschen zu finden.

\section{Zum Begriff der Kontamination}

Viele Kontaminationen haben den Charakter von Versprechern, weil zwei in bestimmter Weise gleichzeitig aktivierte Wörter in das Bewusstsein des Sprechers treten und von beiden Teile „gewinnen“ (das Wasser verdumpft statt verdunstet oder verdampft, Maurer 1933, S. 105). Solche Formen entstehen ohne Absicht und zufällig und sind in der Regel einmalig. Eine andere Art von Verarbeitungsfehlern tritt teils sogar erstaunlich oft auf, $\mathrm{z}$. B. wird regelmäßig aus Hape Kerkeling Herr Hapeling, aus Hilke Elsen Frau Hilsen. Auch hier drängen zwei Wörter gemeinsam ins Bewusstsein.

Davon zu trennen sind absichtliche Bildungen, die spielerisch (hyäßlich aus Hyäne und häßlich), satirisch-ironisch (Sparminator aus sparen und Terminator - Finanzminister Eichel), zum Erregen der Aufmerksamkeit (porNO), als dialektale Übergangserschei- 
nung (Deichsel - Geigsel - Geisel, Maurer 1933, S. 84) oder sprachökonomisch (engl. smog aus smoke und fog für den mit Abgasen und Rauch gemischten Nebel) entstanden sind. Das Phänomen hat mittlerweile einen festen, wenn auch kleinen Platz in den gängigen Wortbildungslehren gefunden (vgl. z.B. Donalies 2002, Erben 2000, Fleischer/Barz 1995, Motsch 1999, Naumann 2000, Simmler 1998, allerdings Eichinger 2000, Eisenberg 2000).

Die Geschichte der Bezeichnung dieser sprachlichen Erscheinung ist abwechslungsreich. Für Maurer gehören die Kreuzungen, die er auch Kontaminationen nennt, zur Wortbildung, weil hier im Gegensatz zur Urschöpfung vorhandener Sprachstoff Verwendung findet (Maurer 1933, S. 13, 95 f.). Er bespricht hauptsächlich Dialektwörter, aber auch Versprecher. Henzen gebraucht Wortverschmelzung, Wortkreuzung und Kontamination parallel und fasst darunter Versprecher und dialektale und damit stabile Wortschatzeinheiten, aber auch Formen wie Kompromissgeburt, Medizyniker, wesentiell und trennt sie von den Zusammensetzungen (Henzen 1957, S. 15 f., 249, 255). Hansen (1963) unterscheidet blending bzw. Wortmischung im eigentlichen Sinne als produktives Wortbildungsmuster (im Englischen) von Fehlleistungen, den Kontaminationen. Windisch (1991) unterteilt seine Wortverschmelzungen in Wortkreuzung und Wortüberschneidung. Ronneberger-Sibold zählt die Wortkreuzung zur Wortschöpfung. Versprecher, die sie wie Hansen und Meid Kontaminationen nennt, schließt sie aus. Schulz (2004) benutzt den Begriff Wortkreuzung, schließt jedoch Versprecher mit ein. Fleischer/Barz (1995, S. 47) sprechen von Wortkreuzung und verweisen im Wesentlichen auf Grésillon. Donalies bezeichnet die Kontamination (auch Wortkreuzung, Wortverschmelzung, Wortmischung) als Wortbildungsart, als Untertyp der Komposition (Donalies 2002, S. $92 \mathrm{f}$.). In Barz (2005) gelten Wortkreuzung, Kontamination und Wortverschmelzung ebenfalls als gleichwertig. Sie grenzt diese Form der peripheren Wortbildung aber von der Komposition ab.

Als Quintessenz wird im vorliegenden Artikel von Kontaminationen gesprochen (auch Wortkreuzungen, Wortverschmelzungen etc.) für unbeabsichtigte als auch für absichtliche Formen. Wie noch gezeigt wird, sind es keine Komposita. Sie stellen einen peripheren, seltenen und in der Regel auffälligen Wortbildungstyp dar und sind nicht immer klar von anderen Wortbildungsmöglichkeiten zu trennen. Der nächste Abschnitt untersucht, wie selten Kontaminationen tatsächlich sind. Anschließend werden Abgrenzungsprobleme anhand von mehr oder weniger schwer klassifizierbaren Beispielen besprochen, um die Ausdehnung der Kategorie aus grammatischer Sicht zu verdeutlichen.

\subsection{Quellen}

Eine der ausführlichsten Untersuchungen überhaupt, die von Almuth Grésillon (1984), bezog sich hauptsächlich auf Heine und damit auf eine literarisch-poetische Sprache eines einzelnen Schriftstellers. Maurers (1933) Schwerpunkt lag bei Dialektbeispielen. Hansen (1963) zog seine amerikanischen Belege aus sprachwissenschaftlichen Veröffentlichungen, Zeitung, Literatur und Wörterbüchern. Ronneberger-Sibold (2005) arbeitet zur Zeit mit Produkt- und Warennamen. Einhellig wird die Meinung vertreten, dass die Bildung von Kontaminationen eine seltene Erscheinung ist, darum werden im Folgenden einige neuere Arbeiten, die Wortbildungsprodukte aus verschiedenen Quellen untersuchen, daraufhin betrachtet. 


\subsubsection{Siebold}

Oliver Siebold (2000) untersucht ca. 4200 Neubildungen in Science Fiction-Texten. Meist handelt es sich um Übersetzungen. Kunstwörter werden bis auf einige Ausnahmen ausgeklammert. Die Sammlung umfasst 57 Kontaminationen $(1,34 \%)$, die er definiert als „Verschränkung bzw. Übereinanderblenden zweier Grundmorpheme oder komplexer Wörter" (Siebold 2000, S. 20). Siebold unterscheidet zwischen einem Ineinderflechten der Komponenten (Schattelit aus Schatten und Satellit, Pfuhlmond aus Pfuhl und Vollmond, Moorzean aus Moor und Ozean, Curdelio aus Kurdel und Fidelio, Papyrokratie aus Papyrus und Bürokratie) und der Übereinstimmung von Kompositagliedern wie in Milchstraßenkreuzer aus Milchstraße und Straßenkreuzer. Hervorzuheben ist dabei, dass die Grenze zwischen Kompositum und Kontamination bzw. zwischen Kontamination und Kunstwort oft nicht klar ist, so dass die Menge an Kontaminationen eher höher liegt, weil Siebold Fälle wie Kontrafessor zu kontra und Professor oder Dreidechse zu drei und Eidechse nicht mit einbezieht. Das relativ verstärkte Aufkommen dieser Wortbildungsmethode dürfte als markant für Science Fiction-Texte gelten, die unbekannte und exotische Dinge mit dem entsprechend klingenden Begriffen benennen.

\subsubsection{Peschel}

Corinna Peschel (2002) untersucht Wortneubildungen der Pressesprache und findet z.B. Waterkantgate aus Waterkant und Watergate als auffallend und witzig, dem folgen Bimbesgate mit Bimbes, Cliquen-Wirtschaft' und Warschau-Gate. Eine weitere Kontamination ist Sparminator aus sparen oder Sparminister und Terminator, weiter Sperminator (besonders leistungsfähiger Samenspender), Lassinator (zu Lasse Kjus, dem Sieger eines Ski-Weltcups). Heym-lich entsteht aus (Stefan) Heym und heimlich, stahlbadern aus Stahl und salbadern. Das Beispiel stammt aus einem Artikel zur Wehrpflicht, was zur Assoziation von Stahl mit Waffen führt. Salbadern mag an viel und nicht unbedingt gehaltvolles Reden denken lassen. Insgesamt handelt es sich um sehr wenig Belege.

\subsubsection{Herberg/Kinne/Steffens}

Dieses Autorenkollektiv (Herberg/Kinne/Steffens 2004) entnahm die 700 neuen Wörter und Phraseologismen, von denen $40 \%$ aus dem Englischen stammen, ebenfalls aus der Pressesprache. Für Herberg et al. sind Kontaminationen Zusammensetzungen: Besserwessi als wortspielerische Verknüpfung von Besserwisser und Wessi, Westalgie (Westen, Nostalgie) gebildet zu Ostalgie (Osten, Nostalgie), sowie Wossi (Wessi, Ossi). Außerdem gibt es die Lehnwörter Edutainment (education, entertainment), Emoticon (emotion, icon), Netizen (Internet, citizen), Infotainment und Netiquette (Internet/network, etiquette). Deutsche Kontaminationen sind also wieder sehr selten.

\subsubsection{Bernard/Heidtmann/Wichmann}

Eine andere Sammlung aus Zeitungstexten, aber für ein breites Publikum gedacht, bieten Bernard, Heidtmann und Wichmann (2006) mit ihren über 600 wichtigsten neuen Wörtern des frühen 21. Jahrhunderts, herausgegeben vom SZ-Magazin. Darunter sind auch Beispiele, die in den letzten Jahren einen Bedeutungswandel durchgemacht haben. Die Lexeme und Phrasen stammen aus der politischen Berichterstattung, dem Jargon der Technik, der Kultur, des Sports und des Boulevards. 
Auch hier gibt es nicht allzu viele Kontaminationen, neben den Fremdwörtern Bollywood (Bombay, Hollywood), governator (governer, terminator), Livestrong (live, Armstrong), Parmalat (ital., Parma, latte), Phishing (password, fishing), Podcast (iPod, broadcast) lediglich Bionade (bio, Limonade), Goleo VI (engl. goal, lat. leo, sp. ole), Sanifair (frz. sanitaire, engl. fair) und Teuro (teuer, Euro).

\subsubsection{Elsen}

In dieser Studie (Elsen 2004) wurden Belege aus verschiedenen Varietäten des Deutschen zusammengetragen. Die der Chemiefachsprache entnommenen Beispiele bezeichnen Substanzen wie Aflatrem, ein Inhaltsstoff von Aspergillus flavus, der zu den tremorgenen Toxinen gehört. Meist jedoch handelt es sich um Produktnamen, die gut speicherbar und griffig sein sollen. Beza-Lande sind Dragees mit Bezafibrat, einer der früheren Hersteller hieß Delalande Arzneimittel GmbH. Centrivac sind Vak.-Konzentratoren mit u.a. Zentrifugal-Verdampfer. Wie die Beispiele zeigen, können also durchaus zwei Wortanfänge kombiniert werden. In der Jugendsprache gab es beispielsweise Videot, Schwachfug, Zeitgeisterfahrer. Bei klaufen aus klauen und kaufen in der Bedeutung ,stehlen' und dammeln aus gammeln und dameln in der Bedeutung ,faulenzen' weist die Kontamination die Bedeutung nur eines der beiden beteiligten Wörter auf.

Axel Hacke bildet die sehr spielerischen Camelopardin, hyäßlich aus Hyäne und häßlich, Schmetterschönling und Schnuppupperle aus Schnupperle und Pupperle, Janosch schnurgeradeaus, Michael Ende Ulkohol, alkohöllisch. Mit gehobenerem Vokabular arbeiten die Zeitungen - Re-Sozialisierung und Anthraxarktis aus Anthrax und Antarktis. Bei den rund 4600 neuen Wörtern, die sich mit jeweils etwa 500 - 700 Belegen auf acht Subkorpora verteilten, gab es 34 (Chemie), 2 (Linguistik, Zeitung), 7 (Jugendsprache), 4 (Literatur) bzw. 33 (Kinderbücher) Kontaminationen. Das heißt, dass diese Art der Wortbildung unterschiedlich stark genutzt wird, was mit der jeweiligen Kommunikationsfunktion zusammenhängen mag. Es bedeutet aber auch, dass Pressetexte als Quellen nicht unbedingt repräsentativ für das Deutsche gelten können - die Kontaminationen bildeten in dieser Studie einen Anteil von 0,39\% in der Pressesprache, 10,92\% bei Michael Ende, 6,73\% in der Chemiesprache bzw. 1,78\% im Gesamtkorpus.

\subsection{Abgrenzungen und Übergangserscheinungen}

Die Kontamination scheint bevorzugt in stilistisch markiertem Rahmen aufzutreten, in Kinderbüchern, in der SF, als werbeträchtige Namen für chemische Produkte oder in kritisch-ironischen Passagen eines Zeitungstextes. Andere Beispiele entstehen in markierten Varietäten. So ist momentan sehr häufig auf den Studenten-Webseiten als beliebte Tätigkeit gruscheln zu finden, was auch zu Gruschel-Mitteilungen führt. Diese Kontamination bedeutet ,grüßen und kuscheln'. Möglicherweise trägt die morphologische Bauweise mit zur stilistischen Prägung bei und stabilisiert die Randposition des Phänomens. Besonders häufig kommen die Kontaminationen aber nicht vor. Trotzdem verdeutlichen die genannten Beispiele ein breites Spektrum an Bildungsmöglichkeiten Milchstraßenkreuzer sieht aus wie ein Kompositum, Curdelio und Aflatrem sind ohne Zusatzinformationen nicht interpretierbar. Die Grenzbereiche zwischen Kontamination und anderen Wortbildungsmöglichkeiten werden deswegen nun genauer betrachtet. 


\subsubsection{Kunstwort}

Kunstwort wird hier synonym mit Wortschöpfung verwendet. Es handelt sich um völlig arbiträre, nichtkomplexe Formen und damit neue Wurzeln (vgl. u. a. Fleischer/Barz 1995, Barz 2005, Elsen 2005a). Häufig zählt die Kontamination grundsätzlich zur Wortschöpfung und nicht zur Wortbildung, und Produktnamen wie Persil aus Perborat und Silikat weisen nur einen anderen Motivations- bzw. Transparenzgrad auf als Maoam oder Fa (Ronneberger-Sibold 2000, S. 96). Kontaminationen sind aber im Gegensatz zu Kunstwörtern nicht völlig willkürlich, obwohl natürlich oft neue Wurzeln entstehen, da die Kontamination keine morphologische Struktur aufweisen müssen. Die Rekonstruierbarkeit von mindestens zwei Ursprungswörtern ist aber ein ausschlaggebendes Kriterium, das ist bei Persil der Fall. Es gibt Bildungen, die aus Teilen eines Lexems und willkürlichen Zusätzen bestehen, sie gehören bereits zu den Wortschöpfungen. Diese Entscheidung mag für manchen bedenklich erscheinen, doch sind eindeutige Klassifizierungen in den Übergangsbereichen nicht möglich. Biosil, Biopren (Zahnfüllmittel) sind Kunstwörter, weil sil und pren keine Bedeutung tragen, im Gegensatz zu Biotuss (Hustensaft), das zu den Kontamination zählt (tussis, lat., 'Husten') (Elsen 2004, S. 38), wie auch Persil.

Weitere Abgrenzungsprobleme ergeben sich bei Kunstwörtern mit klangsymbolischen Endsilben wie Acerbon, Bindol aus der Fachsprache der Chemie und Crescentin, Sykanit, Erosan für fiktive Substanzen (Elsen 2004, 2006).

\subsubsection{Kurzwort}

Mit Persil, Eduscho und Haribo bewegen wir uns in Richtung Kurzwörter (BRD, TÜV, Kripo). Persil ist kein Kurzwort, weil eine Vollform fehlt. Die beteiligten Wörter bilden keine Einheit und sind nicht synonym zu dem neuen Lexem (Greule 1996, S. 200, Kobler-Trill 2002, S. 453). Das gleiche gilt für Eduscho und Haribo, die aus Eduard Schopf bzw. Hans Riegel, Bonn gewonnen werden, aber keine Kurzformvarianten der Namen für die Herren Schopf und Riegel bedeuten wie Lisa zu Elisabeth oder Magda zu Magdalena. Im Unterschied zum Kurzwort, das eine parallele Langform aufweisen muss und mit ihr zunächst einmal bedeutungsgleich ist, wird mit der Kontamination ein neuer Referent erstbenannt, obwohl hier auch ein Kürzungsvorgang beteiligt ist (Kobler-Trill 2002).

\subsubsection{Konfixe}

Im Zusammenhang mit Beispielen wie Infotainment und Topminator wird auch die Möglichkeit von Konfixbildung diskutiert. Barz bespricht Infotainment bei der Kontamination, aber auch bei den Postkonfixen (Barz 2005, S. 678, 691). Genausogut kann es sich um Analogiebildungen zu Kontaminationen wie Tennistainment, Exkreminator, Vitaminator, Ebayminator, Sparminator (Michel 2006) handeln. Da Konfixe Grundmorpheme sind (Fleischer/Barz 1995, S. 25, Glück 2000, S. 362, Elsen 2005a) und eine eigene lexikalische Bedeutung tragen, der Wort,,rest" minator bzw. tainment diese Eigenschaften jedoch nicht aufweist, ist die Interpretation als Kontamination, zumindest momentan, noch plausibler. Ob solche Wortfragmente einmal den Status eines Morphems erhalten, ist zur Zeit offen. Außerdem ist die Entwicklung zum Suffix ebenfalls nicht auszuschließen. 


\subsubsection{Ableitung}

In einem Text von Michael Ende werden in einem Gedicht, das ,in der Fachsprache der Zauberer" verfasst ist, viele lateinisch-wissenschaftlich klingende Wörter gebildet, ohne dass es zu Bedeutungsangaben kommt (Elsen 2004). Krimminol (N.) könnte als Kontamination aus krimminell und Alkohol oder Ähnlichem verstanden werden, aber es gibt kein eindeutiges zweites Wort. Es könnte zu den Ableitungen zählen, aber -ol ist kein Standardmorphem des Deutschen. In der Nomenklatur der Fachsprache der Chemie allerdings verweist -ol auf das Vorhandensein der Hydroxylgruppen, und in Trivialnamen zeigt es den Bestandteil Öl (lat. oleum) an. Daher kann das Wort in der „Fachsprache der Zauberer" als Derivat eingeordnet werden, weil es eine fachsprachliche Endung hat, allerdings nur, weil Bedeutungshinweise fehlen und der Kontext auf eine Fachsprache hindeutet.

Henzen (1957) berichtet von einer Entwicklung von Kontamination zu Suffix, wenn zu einer Bildung Gruppen bedeutungsverwandter neuer Formen entstehen. Er führt zunächst barlatschen aus parlare und blatschen auf (Henzen 1957, S. 252 ff.). Weiter bespricht er den Wortausgang -atschen oder -ätschen in einer neu entstandenen Gruppe von Verben mit der Bedeutungskomponente ,schwatzen' wie ballatschen, polatschen, blatschen, blätschen oder parlatschen. Dieser entwickelt sich zu einem Suffix in weiteren Verben (tratschen, tretschen, tralatschen etc.). Die Kombination von Analogie und erhöhter Frequenz ebnet den Weg von Kontamination zu Derivation, ohne dass eine klare Grenze festzustellen wäre.

Für das Amerikanische stellt Hansen (1963) eine vergleichbare Methode vor, neue Wörter zu bilden, die dort etwas verbreiteter zu sein scheint. Er findet im Abschnitt über Zusammenziehungen, zu denen er auch sexpert aus sex und expert zählt, Produktivität. Ausgehend von einer Form wie walkathon zu walking marathon werden weitere ähnliche Beispiele geprägt, bis die ursprünglich als Wortfragment verwendete Komponente ein Eigenleben entwickelt und als Affix gilt. Im Englischen gibt es mittlerweile -teria, -gate, -(o)holic, -burger oder -buster mit Affixstatus (Fradin 2000, Szymanek 2005). In der Übergangsphase kann nicht zwischen Wortzusammenziehungen und Ableitungen geschieden werden. Eine aktuelle Entwicklung bei uns, deren Beginn wohl auf Entlehnungen komplexer Wörter zurückzuführen ist, bevor es zu Kontamination im Deutschen kam, betrifft die minator- und ainment-Bildungen. Für sie muss sich eine solche Verselbständigung der auf Fehlsegmentierung basierenden Einheiten jedoch erst noch zeigen.

\subsubsection{Kompositum}

Für viele, z. B. Herberg et al. (2004) oder Donalies (2002), sind Kontaminationen Zusammensetzungen. Auch wenn beides einige Gemeinsamkeiten aufweist, z. B. die Beteiligung zweier Lexeme und oft auch ein determinierendes oder kopulatives Verhältnis der Bedeutungen, gibt es wichtige Unterschiede. Hansen betont, dass die Bedeutung der Kontamination nicht einer einfachen Addition der Bedeutung der beteiligten Lexeme entspricht. Außerdem werden nicht Wörter bzw. Wurzeln, sondern Wortteile verbunden, in dem sie ineinander greifen oder aufeinander folgen (Henzen 1957, S. 249).

Wie bereits in Elsen (2004, S. 38 f.) ausgeführt wurde, ist manchmal die Abgrenzung von Kontamination und Kompositum schwierig. Sind zwei Wurzeln vorhanden und ist 
der Ausdruck semantisch motiviert, liegt Komposition vor, beispielsweise bei Aaptamin, einem Amin mit einem Bestandteil des Schwammes Aaptos aaptos. Sind jedoch lediglich Wortfragmente erkennbar, so dass die morphologische Struktur der Geberwörter verletzt wird, und bleiben außerdem von den semantischen Komponenten nur noch einzelne übrig, weil lediglich bestimmte Assoziationen ausgelöst werden sollen, zählt ein Wort nicht mehr zu den Zusammensetzungen. Die zweite Einschränkung ist nötig wegen Beispielen wie Biotuss, für die eine Interpretation als (Determinativ-, Kopulativ-) Kompositum oder Metapher nicht gerechtfertigt ist.

Siebold führt weitere schwer klassifizierbare Beispiele auf, zu denen ihm Bedeutungshinweise fehlen. So zählt er Dreidechse zu den Analogiebildungen als Übergang zu den Komposita, nicht zu den Kontaminationen. Fäulnickel kann er nicht interpretieren, denn Fäule und Nickel sind zwar erkennbar, passen aber nicht zur Bedeutung der neuen Form, die eine pejorative Bezeichnung für Bewohner eines fremden Planeten sind, die in anderen Lebewesen leben.

Grundsätzlich sind auch Formen wie Pfuhlmond (Siebold 2000, S. 81) als problematisch zu sehen, da sie zwar durch eine Vermischung zweier Wörter, hier Pfuhl und Vollmond, entstanden sein mögen, trotzdem aber Komposita sind. Denn auch die Kombination von Pfuhl und Mond analogisch zu voll und Mond ist ja durchaus als Entstehungshintergrund denkbar. Windisch trennt solche haplologen Wortüberschneidungen von der Kreuzung (Himmelsschlüsselbein vs. Phrasnost aus Phrase(n) und Glasnost (Windisch 1991, S. 36). Oft können ohne inhaltliche oder textuelle Informationen keine Entscheidungen getroffen werden, denn wie Schmid betont muss bei einem Lexem wie Schweißheilige von einer Kontamination ausgegangen werden wegen des Rückbezugs auf Schweiß und Eisheilige (Schmid 2003, S. 266, 270). Das ist aber nur bei semantischer Durchsichtigkeit und/oder durch eindeutige textuelle Hinweise möglich. Grèsillon (1984) räumt ein, dass eine kleine Gruppe an Beispielen wie etwa Rotköpfchen sowohl als Kontamination als auch als Komposition einstufbar ist, je nach Kontext. Bei Formen wie Himmelsschlüsselbein oder Schokoladentisch, die Windisch bzw. Grésillon erwähnen, ist das nicht der Fall. Am extremsten dürfte die Problematik in Grésillons Primadonna deutlich werden, das sie auf Primadonna und Madonna zurückführt (Grésillon 1984, S. 41, 46). Sie zieht den Schluss ,que la frontière entre composition/dérivation d'une part et motvalise d'autre part ne se situe pas nécessairement en un point fixe, mais sur un parcours marqué encore par l'analogie et déjá par la formation-valise“"(Grésillon 1984, S. 51).

\subsection{Typen}

Die bisherigen Betrachtungen zeigen nun zweierlei. Erstens vereint die Kategorie Kontamination ein breitgestreutes Spektrum an Gestaltungsmöglichkeiten. Zweitens stellt sie eine seltene, jedoch nicht konsequent genutzte Wortbildungsmöglichkeit dar. Das betont den stilistischen Charakter dieses Wortbildungstyps. Es wird nun zunächst der erste Punkt weiter verfolgt, indem Strukturierungsmöglichkeiten ausgelotet werden.

Um Typologien waren die Autoren seit jeher bemüht. Die Vorschläge reichen von zwei - alle zugrundeliegenden Wörter werden gekürzt oder nur eines (Wentworth 1934 in Cannon 2000, S. 954) - bis zehn und mehr Unterarten (Schmid 2003, Fradin 2000). Formal betrachtet gibt es längere oder kürzere homophone Wort(teil)e (Waterkantgate, Müllionärin, Dollarubel), Trunkation (Persil, Tomoffel), beides (Komplikatesse aus Komplikation und Delikatesse) oder die seltene Einfassung (enchâssement) vgl. frz. expojarrysi- 
tion aus exposition und Jarry (Grésillon 1984, S. 24 f.). Das heißt, Wortteile verbinden sich und/oder es kommt zu Überlappungen, seltener zu einer Integration eines Wort in ein anderes, was dann außerdem orthographisch sichtbar gemacht werden kann (porNO). Die semantischen Typen sind noch nicht gezählt, Schmid (2003) führt die zehn häufigsten an. Zwar handelt es sich oft um Kopulativ- oder, eher noch, um Determinativverhältnisse, es kommt jedoch eine Verschlüsselung als Besonderheit hinzu, denn dic Sprachbenutzer verwenden ja Watergate und gerade nicht Waterkantwatergate (Peschel 2002, S. 177). Die Kontamination gehört meistens in die gleiche Wortklasse wie die Ausgangswörter. Außerdem weisen die beteiligten Wörter meist ähnliche, auch gegensätzliche Bedeutungen auf (Maurer 1933, S. 108, Grésillon 1984, S. 26 f.), dies ist aber nicht zwingend nötig (Fleischer/Barz 1995, S. 47). „Most blends come from semantically related etyma that convey some of their meaning onto the blend, and the most commercially successful blends are often also morphologically transparent" (Cannon 2000, S. 954). Ein wenig transparentes Beispiel ist Novasil, das Silber enthalten soll, ohne dass der Ausdruck interpretierbar wäre (Ronneberger-Sibold 2005, S. 219). Ist ein Ausdruck nicht gleich verständlich, so mag Absicht zugrunde liegen, um Leserneugier zu wecken (Dittgen 1989, S. 110 ff., Poethe 2002), oder die Bildung ist schlecht gelungen, wenn sie nicht dechiffrierbar ist. Häufig ist eine gewisse Offenheit der Interpretationsmöglichkeiten beabsichtigt, um Aussagen vage zu halten und um Repressalien, die bei einer eindeutigen Behauptung drohen, zu umgehen, wie in der Zeitungssprache. Insgesamt gibt es also einfache und schwierige Fälle von Kontamination.

Wie auch Schulz (2004, S. 300) schlussfolgert, sind die Regularitäten abgestuft, die Transparenz ist graduell unterschiedlich. Statt nun eine allgemeingültige Definition zu formulieren, die die Kontamination deutlich von den anderen Möglichkeiten der Wortbildung abhebt, wird der aus den bisherigen Textabschnitten gefilterte Prototyp beschrieben. Das ermöglicht uns außerdem, die Gruppe der Kontaminationen zu gliedern. Eindeutige Beispiele sind typische bzw. zentrale Vertreter, denn sie weisen die wichtigsten bzw. häufigsten bzw. die repräsentativen Eigenschaften auf. Periphere Vertreter weisen weniger Eigenschaften auf. Manche peripheren Vertreter können auch keine gemeinsamen Merkmale aufweisen. Übergänge zwischen Kategorien sind fließend, so dass es zu nicht eindeutig klassifizierbaren Beispielen kommt.

\section{Eine prototypische Betrachtungsweise - Möglichkeiten und Grenzen}

Die prototypische Kontamination, z. B. wesentiell aus wesentlich und essentiell, ist absichtlich geschaffen. ${ }^{1}$ Sie ist transparent bzw. begrifflich motiviert. Sie geht auf zwei Ausgangslexeme zurück. Es gibt von einem Ursprungswort einen Anfangsteil, vom anderen einen Endteil, beide gehören zu einer Wortklasse, sind bedeutungsverwandt (bis hier vgl. Hansen 1963, S. 123) und stammen aus dem usuellen Lexikon. Bedeutungsaspekte der Einzellexeme finden sich im Gesamtausdruck wieder. Die Kontamination ist rekonstruierbar und stilistisch gefärbt.

Für die Gruppierung der Beispiele innerhalb der Kategorie ist eine Gewichtung der Merkmale nötig, die hier nicht geleistet werden kann, aber es können Vorschläge gemacht werden. Zwei Lexeme als Basis ist ein so markantes Charakteristikum, dass es oft

\footnotetext{
${ }^{1}$ Die Eigenschaften sind lediglich aufgezählt, ohne damit eine Gewichtung zu implizieren.
} 
als allgemeingültig angenommen wird (z. B. Schulz 2004), obwohl auch seltene Fälle von drei Ursprungswörtern auftreten. Die Verwendung ausschließlich mit Wortteilen dürfte hingegen kein sehr wichtiges Kennzeichen sein, die Rekonstruierbarkeit wiederum schon. Diese hängt freilich von der Erkennbarkeit mindestens eines der beteiligten Lexeme ab (Schulz 2004, S. 301). Schulz (2004) fand beispielsweise als häufigstes Kriterium, dass mindestens ein Ausgangslexem graphisch oder lautlich vollständig erhalten bleibt. Dies mag mit seiner Wortsammlung zusammenhängen, die sich hauptsächlich auf die Beispiele der zu den Kontaminationen veröffentlichten Fachartikel beruft. Die Frequenz jedenfalls ist sicherlich ein bedeutender Faktor. ${ }^{2}$ Um ein anderes wichtiges Moment dürfte es sich bei der Plakativität des Ausdrucks handeln. Sie kann jedoch nur über ausgedehnte Rezipientenbefragungen näher bestimmt werden. Ein Vorschlag für die Anordnung der Kontaminationen in der Kategorie und in Bezug auf andere Kategorien ist in Abbildung 1 dargestellt mit verschiedenen im Text erwähnten Beispielen.

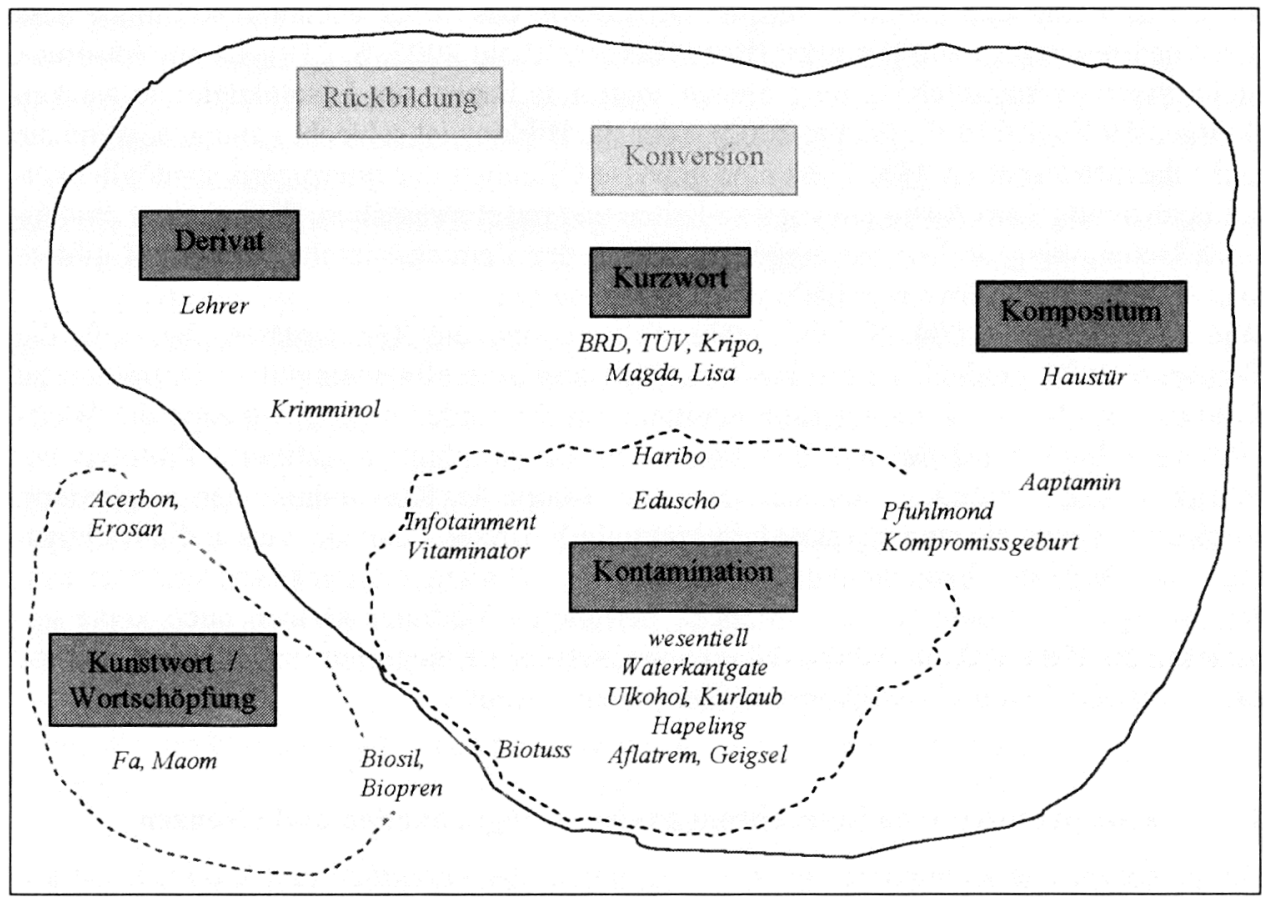

Abbildung 1: Kontaminationen innerhalb der Kategorie und im Bezug auf andere Wortarten

Wesentiell ist ein gutes Beispiel, weil es alle oben genannten Eigenschaften besitzt. Etwas weniger typisch ist Waterkantgate, weil ein Wort komplett erhalten ist, Kurlaub, weil die Bildung stilistisch nicht gefärbt ist, Ulkohol, Westalgie, weil die Ursprungswörter semantisch nichts miteinander zu tun haben, alkohöllisch, weil mit verschiedenen Wortklassen gearbeitet wird. Weniger typisch sind Hapeling, da es sich nicht um eine absichtliche Bildung handelt, Novasil, Aflatrem, weil nicht sehr transparent, Geigsel,

${ }^{2}$ Ob Häufigkeit Grund oder Anzeichen für Prototypikalität ist, sei hier dahin gestellt (vgl. Taylor 1995, S. 52) 
weil nicht aus dem usuellen Lexikon des Deutschen stammend, klaufen, weil nur die Semantik eines Lexems erhalten bleibt. Noch weniger typisch ist Haribo, weil drei Wörter beteiligt sind und drei Wortanfänge kombiniert werden. Pfuhlmond ist ein schlechtes Beispiel, weil es genauso gut ein Kompositum sein kann. Primadonna ist identisch mit einem etablierten Lexem der deutschen Standardsprache, deswegen ist es bereits keine Kontamination mehr. Binsil ist besonders schlecht, weil es nicht transparent ist urd einen Kunstwortanteil besitzt, die Gesamtsemantik hat nur entfernt etwas mit der erkennbaren Komponente zu tun, es ist schon keine Kontamination mehr, sondern ein Kunstwort, allerdings auch kein gutes.

\section{Ausblick}

Bei der Kontamination handelt es sich um eine besondere, eher periphere Wortbildungserscheinung, die keinen eindeutigen und durchgängigen formalen Regeln gehorcht und fast immer zu stilistisch markierten Wörtern und meist auch Okkasionalismen führt, im Gegensatz zum Englischen, das des öfteren stabile Lexeme produziert wie brunch, smog, motel, quasar, liger. Die Belegdichte ist insgesamt niedrig. Beispiele ohne stilistisch gefärbten Hintergrund sind im Deutschen kaum zu finden. Verschiedentlich wurde nach einem System gesucht, das hinter den Bildungen stecken könnte. Die internen Systematisierungsmöglichkeiten reichen jedoch nicht immer aus, um alle Beispiele zu erfassen, sie erfordern einen erweiterten Blickwinkel. Das heißt, eine Bildung kann zusätzlich von außen, von Text- und Gebrauchsbedingung aus betrachtet werden oder vom Endprodukt her (vgl. auch Ronneberger-Sibold 2005), von der Gestalt aus. Die Kontamination wird nämlich gerade dann verwendet, wenn die üblichen Wortbildungsverfahren nicht mehr genügen - um stilistische, spielerische, sarkastische Intentionen zu verwirklichen, um indirekte Aussagen zu treffen, um zu einem bestimmten Klangbild mit den entsprechenden Assoziationen zu gelangen. Offenbar spielt wie bei den Kunstwörtern das gesamte Erscheinungsbild eine Rolle, so dass morphologische Entstehungsweisen und strukturelle Erwägungen in den Hintergrund treten.

Bereits bei der Untersuchung zum Erwerb von Wörtern zeigte sich, dass Kinder bei dem Versuch, Wörter auszusprechen, teilweise stark abweichende oder kaum wiedererkennbare Lautkörper produzieren, die aber nicht willkürlich sind. Leider sind solche Beispiele in der Literatur weniger häufig zu finden, denn schlecht zu transkribierende Äußerungen bleiben bei den meisten Datenerhebung unberücksichtigt. Es zeigte sich, dass in einer frühen Phase Probleme bei komplexen Wörtern auftreten. Es werden nicht alle suprasegmentalen und phonetischen Informationen gemeinsam verarbeitet. Die Kinder geben den prosodischen Merkmalen den Vorrang. Die Anzahl der Silben und die Lage des Wortakzentes werden am sichersten verarbeitet. Dann sind offenbar die Vokale der haupttonigen Silbe erreichbar. Dass Silbenmenge und Akzentlage gut erkennbar sind, zeigen die verschiedenen Ersatzstrategien, die verwendet werden, um genau diese sprachliche Information wiederzugeben (Elsen 1999, 2000, in Vorb. a). Bei tongue-ofthe-tip-Experimenten waren Silbenzahl und Betonungsmuster diejenigen lautlichen Merkmale, an die am sichersten erinnert wurde (Aitchison 1987, S. 123 f.). Und erstaunliche Parallelen fanden Schulz (2004) und Ronneberger-Sibold (2005) bei der Betrachtung einiger Kontaminationen. Auch hier dominierten Silbenzahl und Lage des Wortakzentes, was zur Ermittlung des damit dominanten Wortes führte. Offenbar ist das Klang- 
bild eines Ausdrucks entscheidend und nicht der morphologische Weg, der dort hinführt. Dies wurde in Untersuchungen der Werbesprache (Ronneberger-Sibold 2000, 2005), in der Chemiefachsprache und in Kinderbüchern (Elsen 2004) und bei der Namengebung für phantastische Gestalten (Elsen 2005a, 2006, 2007a, b, 2008, in Vorb. a, b) bereits mehrfach konstatiert. Je nach Bedarf werden bei einem Wort über das Klangbild bestimmte Assoziationen hervorgerufen. Namen von phantastischen Wesen sollen fremd oder bekannt, gutmütig oder gefährlich klingen. Heines Wörter sollen satirisch wirken, Markennamen wiederum exotisch. Entsprechend erhält Ronneberger-Sibold unterschiedliche Gewichtungen der Transparenzgrade. Und natürlich ist es dann auch nicht verwunderlich, wenn in den verschiedenen Korpora die Kontamination unterschiedlich intensiv genutzt wird. Der vorliegende Untersuchungsbereich betrifft die Parole. Darum wird als zusätzliches Bearbeitungskriterium neben den (proto)typischen Kennzeichen auch die durch die kreativen Fähigkeiten und den kommunikativen Bedürfnissen abhängige Gestalt eines Ausdrucks relevant. Aus Sicht des Sprachwissenschaftlers bestimmt der Sprachbenutzer über einen längeren Zeitraum hin, was gut ist oder was nicht und trägt zu Gewichtsverschiebungen im Kriterienbündel und letztendlich zum Sprachwandel bei. Aus Sicht des Sprachbenutzers dient die Kontamination, zusammen mit den Kunstwörtern, in den allermeisten Fällen als assoziationsträchtiges Ausdrucksmittel, das gerade nicht klare und eindeutige Referenten benennt und eine Lücke in der Gesamtgrammatik füllt.

\section{Literatur}

Aitchison, Jean (1987): Words in the Mind. An Introduction to the Mental Lexicon. Oxford.

Barz, Irmhild (2005): Die Wortbildung. In: Duden Band 4. Die Grammatik, 7. Auflage. Mannheim/Leipzig/ Wien/Zürich. S. 641-772.

Bernard, Andreas/Heidtmann, Jan/Wichmann, Dominik (2006): Sprechen Sie Gegenwart? Lexikon des frühen 21. Jahrhunderts. Aktualisierte und erweiterte Taschenbuchausgabe. München.

Cannon, Garland (2000): Blending. In: Booij, G./Lehmann, C./Mugdan, J.: Morphologie. Ein internationales Handbuch zur Flexion und Wortbildung. Berlin/New York. S. 952-956.

Carroll, Lewis (1976): Complete Works, with an Introduction by Alexander Woollcott and Illustrations by John Tenniel. New York.

Dittgen, Andrea Maria (1989): Regeln für Abweichungen. Funktionale sprachspielerische Abweichungen in Zeitungsüberschriften, Werbeschlagzeilen, Werbeslogans, Wandsprüchen und Titeln. Frankfurt/M. et al.

Donalies, Elke (2002): Die Wortbildung des Deutschen. Tübingen.

Eichinger, Ludwig M. (2000): Deutsche Wortbildung. Eine Einführung. Tübingen.

Eisenberg, Peter (2000): Grundriß der deutschen Grammatik I. Das Wort., Korrigierter Nachdruck. Stuttgart/Weimar.

Elsen, Hilke (1999): Ansätze zu einer funktionalistisch-kognitiven Grammatik. Tübingen.

Elsen, Hilke (2000): Das Verhältnis zwischen segmentalen und suprasegmentalen Merkmalen in der Kindersprache. In: Germanistyka 15, S. 49-70.

Elsen, Hilke (2004): Neologismen. Formen und Funktionen neuer Wörter in verschiedenen Varietäten des Deutschen. Tübingen. 
Elsen, Hilke (2005a): Das Kunstwort. In: Muttersprache 115, S. 142-149.

Elsen, Hilke (2005b): Deutsche Konfixe. In: Deutsche Sprache 33, S. 133-140.

Elsen, Hilke (2006): Pseudomorpheme. Fiktive Namen im Übergangsbereich von Phonologie und Morphologie. In: Muttersprache 3, S. 242-248.

Elsen, Hilke (2007a): Gestaltverarbeitung. In: Deutsch als Fremdsprache 44, S. 162-165.

Elsen, Hilke (2007b): Die Wortbildung der Eigennamen in fiktionalen Texten. In: Zeitschrift für Literaturwissenschaft und Linguistik 148, S. 184-197.

Elsen, Hilke (2008): Phantastische Namen. Die Namen in Science Fiction und Fantasy zwischen Arbitrarität und Wortbildung. Tübingen.

Elsen, Hilke (in Vorb. a): Between phonology and morphology. In: Michel, S./Onysko, A. (Hg.): Cognitive Approaches to Word Formation. Berlin/New York.

Elsen, Hilke (in Vorb. b): Grenzgänger. Unschärfe und Typikalität bei Namen aus Science Fiction und Fantasy. In: Michel, S./Toth, J. (Hg.): Wortbildungssemantik zwischen Language und Parole. Semantische Produktions- und Verarbeitungsprozesse komplexer Wörter. Stuttgart.

Elsen, Hilke/Michel, Sascha (2007): Wortbildung und Sprachgebrauch. Desiderate und Perspektiven einer etablierten Forschungsrichtung. In: Muttersprache 117, S. 1-16.

Erben, Johannes (2000): Einführung in die deutsche Wortbildungslehre. 4. Auflage. Berlin.

Fleischer, Wolfgang/Barz, Irmhild (1995): Wortbildung der deutschen Gegenwartssprache, 2. Auflage. Tübingen.

Fradin, Bernard (2000): Combining forms, blends and related phenomena. In: Doleschal, U./Thornton, A. M. (Hg.): Extragrammatical and Marginal Morphology. München. S. 11-59.

Glück, Helmut (2000): Metzler Lexikon Sprache, 2. Auflage. Stuttgart/Weimar.

Grésillon, Almuth (1984): La règle et le monstre: le mot-valise. Interrogations sur la langue, à partir d'un corpus de Heinrich Heine. Tübingen.

Greule, Albrecht (1996): Reduktion als Wortbildungsprozess der deutschen Sprache. In: Muttersprache 106, S. 194-203.

Hansen, Klaus (1963): Wortverschmelzungen. In: Zeitschrift für Anglistik und Amerikanistik 11, S. 117142.

Henzen, Walter (1957): Deutsche Wortbildung, 2. Auflage. Tübingen.

Herberg, Dieter/Kinne, Michael/Steffens, Doris (2004): Neuer Wortschatz. Neologismen der 90er Jahre im Deutschen. Berlin/New York.

Kobler-Trill, Dorothea (2002): Die Formseite der Abkürzungen und Kurzwörter. In: Cruse, D. A./ Hundsnurscher, F./Job, M./Lutzeier, P.R. (Hg.): Lexikologie. Ein internationales Handbuch zur Natur und Struktur von Wörtern und Wortschätzen I. Berlin/New York. S. 452-456.

Lehrer, Adrienne (1996): Identifying and interpreting blends: An experimental approach. In: Cognitive Linguistics 7-4, S. 359-390.

Maurer, Friedrich (1933): Volkssprache. Abhandlungen über Mundarten und Volkskunde. Zugleich eine Einführung in die neueren Forschungsweisen. Erlangen.

Meid, Wolfgang (1977): Beziehungen zwischen äußerer und innerer Sprachform: verschränkte Zeichen und fusionierte Inhalte. In: Veröffentlichungen der Kommission für Linguistik und Kommunikationsforschung 6, S. 294-304.

Michel, Sascha (2006): Vom Terminator zum TORminator. Die Wortbildungseinheit -minator: Strukturelle und sozio-pragmatische Analysen. In: Muttersprache 4, S. 289-307.

Motsch, Wolfgang (1999): Deutsche Wortbildung in Grundzügen. Berlin/New York. 
Naumann, Bernd (2000). Einführung in die Wortbildungslehre des Deutschen. Tübingen.

Peschel, Corinna (2002): Zum Zusammenhang von Wortneubildung und Textkonstitution. Tübingen.

Poethe, Hannelore (2002): Wort(bildungs)spiele. In: Barz, I./Fix, U./Lerchner, G. (Hg.): Das Wort in Text und Wörterbuch. Stuttgart/Leipzig. S. 23-40.

Ronneberger-Sibold, Elke (2000): Creative competence at work: the creation of partial motivation in German trade names. In: Doleschal, U./Thornton, A.M. (Hg.): Extragrammatical and Marginal Morphology. München. S. 85-105.

Ronneberger-Sibold, Elke (2005): Zur Definition und Typologie von Wortkreuzungen. Ein Vorschlag auf der Grundlage ihrer relativen Transparenz. In: Fenk-Oczlon, G./Winkler, C. (Hg.): Sprache und Natürlichkeit. Gedenkband für Willi Mayerthaler. Tübingen. S. 205-224.

Schmid, Hans Ulrich (2003): Zölibazis Lustballon. Wortverschmelzungen in der deutschen Gegenwartssprache. In: Muttersprache 3, S. 265-278.

Schulz, Matthias (2004): Jein, Fortschrott und Ehrgeizhals. Wortkreuzungen in der deutschen Gegenwartssprache. In: Zeitschrift für germanistische Linguistik 32, S. 286-306.

Siebold, Oliver (2000): Wort - Genre - Text. Wortneubildungen in der Science Fiction. Tübingen.

Simmler, Franz (1998): Morphologie des Deutschen. Flexions- und Wortbildungsmorphologie. Berlin.

Szymanek, Bogdan (2005): The latest trends in English word-formation. In: Stekauer, P./Lieber, R. (Hg.): Handbook of Word-Formation. Dordrecht.

Taylor, John R. (1995): Linguistic Categorization. Prototypes in Linguistic Theory. 2. Auflage. Oxford.

Windisch, Rudolf (1991): Die Wortverschmelzung - ein ,abscheußliches Monstrum' der französischen und deutschen Wortbildung? In: Romanistisches Jahrbuch 42, S. 34-51.

PD Dr. Hilke Elsen

Ludwig-Maximilians-Universität München

Institut für Deutsche Philologie

Schellingstr. 3 RG

D-80799 München

Email: hilkee@Irz.uni-muenchen.de 